\title{
Mg-Si-Sn 基热电器件电极材料的优化选择与连接工艺
}

\author{
陈 耿 $^{1,2}$, 刘桃香 ${ }^{1,2}$, 唐新峰 ${ }^{2}$, 苏贤礼 ${ }^{2}$, 㓰永高 ${ }^{2}$ \\ (武汉理工大学 1. 材料科学与工程学院; 2. 材料复合新技术国家重点实验室, 武汉 430070)
}

摘 要: 研究了采用不同放电等离子烧结(SPS)工艺获得的单质金属 $(\mathrm{Ni} 、 \mathrm{Cu} 、 \mathrm{Ag} 、 \mathrm{Al})$ 电极与 $\mathrm{Mg}-\mathrm{Si}-\mathrm{Sn}$ 基热电材料 结合界面的微观形貌和成分分布特征, 测试了合金 $(\mathrm{Ni}-\mathrm{Al} 、 \mathrm{Cu}-\mathrm{Al})$ 、金属/合金复合电极材料的热膨胀系数、电导率 和热导率等物性参数。实验结果表明：通过 SPS 烧结可以有效实现电极材料与 Mg-Si-Sn 基材料的连接，复合电极 材料 $\mathrm{Ni}-\mathrm{Al} / \mathrm{Al}(60: 40)$ 和 $\mathrm{Cu}-\mathrm{Al} / \mathrm{Cu}(45: 55)$ 具有高的电导率和热导率, 并且热膨胀系数与 $\mathrm{Mg}-\mathrm{Si}-\mathrm{Sn}$ 基热电材料相匹配, 有可能成为 Mg-Si-Sn 基材料的较理想电极材料。

关 键 词: 镁硅锡; 热电材料; 电极材料

中图分类号: TB34 文献标识码: A

\section{Optimization of Electrode Material and Connecting Process for Mg-Si-Sn Based Thermoelectric Device}

\author{
CHEN Geng ${ }^{1,2}$, LIU Tao-Xiang ${ }^{1,2}$, TANG Xin-Feng ${ }^{2}$, SU Xian-Li ${ }^{2}$, YAN Yong-Gao ${ }^{2}$ \\ (1. School of materials science and engineering, Wuhan University of Technology, Wuhan 430070, China; 2. State Key Labo- \\ ratory of Advanced Technology for Materials Synthesis and Processing, Wuhan University of Technology, Wuhan 430070, \\ China)
}

\begin{abstract}
Metal (Ni, Cu, Ag, Al) electrode was connected with Mg-Si-Sn based thermoelectric materials by Spark Plasma Sintering (SPS) process. Microstructure of interface, sintering process and transport properties, including electrical conductivity, thermal conductivity and thermal expansion coefficient, of electrode materials were investigated. The results showed that composites of $\mathrm{Ni}-\mathrm{Al} / \mathrm{Al}(60: 40)$ and $\mathrm{Cu}-\mathrm{Al} / \mathrm{Cu}$ (45:55) displayed high electrical conductivity, high thermal conductivity and suitable thermal expansion coefficient to $\mathrm{Mg}$-Si-Sn based materials. It is indicated that the two composites have potential to be used as good electrode materials for, and to be connected by SPS sintering to, $\mathrm{Mg}-\mathrm{Si}$-Sn based materials.
\end{abstract}

Key words: $\mathrm{Mg}-\mathrm{Si}-\mathrm{Sn}$; thermoelectric material; electrode material

半导体材料能获得的最大热电优值可通过热电 性能化指标 $\beta=\left(m^{*}\right)^{3 / 2} \mu / \kappa_{\mathrm{ph}}$ 来预测, 其中 $m^{*}$ 为载流 子有效质量, $\mu$ 为载流子迁移率, $\kappa_{\mathrm{ph}}$ 为晶格热电率。 据此计算, $\mathrm{Mg}_{2} \mathrm{X}(\mathrm{X}=\mathrm{Si} 、 \mathrm{Ge} 、 \mathrm{Sn})$ 基化合物的热电性 能化指标 $\beta$ 在 3.7 $14^{[1]}$ 的范围内, 表明这类化合物
具有潜在优异的热电性能, 并具有比重小、熔点高、 原材料储量丰富、价格低廉且组成元素无毒等优点, 所以备受国际上热电研究者的关注。由于 $\mathrm{Mg}_{2} \mathrm{X}$ 基 三元固溶体的晶格热导率远低于二元化合物, 所以 人们主要通过固溶合金化降低材料的晶格热导率,

收稿日期：2014-12-01; 收到修改稿日期：2015-02-04

基金项目：国家重大基础研究计划 973 项目(2013CB632502); 国家自然科学基金(51402222, 51172174, 51401153); 国家 111 计划(B07040)

National Basic Research Program of China (973 Program) (2013CB632502); National Natural Science Foundation of China (51402222, 51172174, 51401153); 111 Project of China (B07040)

作者简介：陈 耿(1987-), 男, 硕士研究生. E-mail: chengeng17@126.com

通讯作者: 刘桃香, 副教授.E-mail: cxzwut@126.com; 唐新峰，教授.E-mail: tangxf@whut.edu.cn 
以优化其热电性能 ${ }^{[2-10]}$ 。Zaitsev 等 ${ }^{[3]}$ 通过对 $\mathrm{Mg}_{2} \mathrm{~B}^{\mathrm{IV}}$ $\left(\mathrm{B}^{\mathrm{IV}}=\mathrm{Si} 、 \mathrm{Ge} 、 \mathrm{Sn}\right)$ 体系热电性能的研究发现, 其中的 $\mathrm{Mg}_{2} \mathrm{Si}_{1-x} \mathrm{Sn}_{x}$ 最具热电应用前景。而 $\mathrm{n}$ 型 $\mathrm{Mg}_{2} \mathrm{Si}_{1-x} \mathrm{Sn}_{x}$ $(x=0.6 \sim 0.7)$ 基化合物由于其重导带与轻导带的简并, 在费米能级附近具有更高的电子态密度，在 700 $750 \mathrm{~K}$ 温度范围内 $Z T$ 值可达 1.3 左右 ${ }^{[9]}$, 是 $\mathrm{Mg}_{2} \mathrm{X}$ 基固溶体中性能最佳者。

然而, 热电材料的商业化应用还离不开热电器 件的研究开发, 目前 $\mathrm{Mg}_{2} \mathrm{Si}$ 基化合物热电器件的研 究主要针对掺杂的 $\mathrm{n}$ 型 $\mathrm{Mg}_{2} \mathrm{Si}$ 材料 ${ }^{[11-16]}$, 其涉及到 的电极材料多采用金属单质 $\mathrm{Ni}$, 因为其热膨胀系数 与 $\mathrm{Mg}_{2} \mathrm{Si}$ 热电材料十分接近, 而且化学性质稳定。 但 $\mathrm{n}$ 型 $\mathrm{Mg}_{2} \mathrm{Si}$ 材料在 $800 \mathrm{~K}$ 下 $Z T$ 约为 $0.6^{[12]}$ ，与性 能优异的 $\mathrm{Mg}_{2} \mathrm{Si}_{1-} \mathrm{Sn}_{x}$ 体系相比, 热电性能相对较低, 因此需要探索出适合 $\mathrm{Mg}_{2} \mathrm{Si}_{1-x} \mathrm{Sn}_{x}$ 高效热电材料固 溶体合金的电极材料。

好的电极材料必须具备高的电导率和热导率、良 好的化学和高温稳定性、热膨胀系数与热电材料相匹 配、与热电材料结合界面的接触电阻与接触热阻低等 特点 ${ }^{[17]}$ 。而符合这些要求又与 $\mathrm{Mg}-\mathrm{Si}-\mathrm{Sn}$ 基热电材料 相匹配的电极材料的探索, 至今尚未见相关报道。本 研究以 $\left(\mathrm{Mg}_{2.16}\left(\mathrm{Si}_{0.3} \mathrm{Sn}_{0.7}\right)_{0.98} \mathrm{Sb}_{0.02}\right)$ 作为 $\mathrm{Mg}$-Si-Sn 基热 电材料, 采用金属单质 $\mathrm{Al} 、 \mathrm{Ni} 、 \mathrm{Cu}$ 和 $\mathrm{Ag}$ 作为电极材 料, 探索其与 $\mathrm{Mg}-\mathrm{Si}-\mathrm{Sn}$ 基热电材料的连接工艺; 首次 尝试采用金属/合金复合物作为电极材料, 探索一系 列不同比例的金属/合金复合物电极材料对基本物性 参数(热膨胀系数、电导率和热导率等)的影响规律, 以 进一步调节电极材料的热膨胀系数, 从而确定适合于 $\mathrm{Mg}-\mathrm{Si}-\mathrm{Sn}$ 基材料的复合电极组成。

\section{1 实验方法}

采用两步固相反应法结合放电等离子烧结技术 制备 $\mathrm{Mg}-\mathrm{Si}-\mathrm{Sn}$ 基热电材料 ${ }^{[10]}$ 。将高纯的 $\mathrm{Mg}$ 粉 (99.9\%)、Si 粉(99.99\%)、 Sn 粉(99.9\%)和 Sb 粉(99.99\%) 按 $\mathrm{Mg}_{2.16}\left(\mathrm{Si}_{0.3} \mathrm{Sn}_{0.7}\right)_{0.98} \mathrm{Sb}_{0.02}$ 化学计量称量混合均匀压 制成块体, 在 $873 \mathrm{~K}$ 进行固相反应, 得到的初始产 物经磨细后压制成块体再在 $973 \mathrm{~K}$ 进行固相反应, 得到的产物再次进行破碎研磨成粉体。电极与热电 材料的连接采用两种不同的 SPS 烧结工艺, 一种是 一步 SPS 烧结法: 首先将混合均匀且过 $37 \mu \mathrm{m}$ (400 目)篮的电极材料粉末置于石墨模具中压平，再将 固相反应法合成的单相 $\mathrm{Mg}_{2.16}\left(\mathrm{Si}_{0.3} \mathrm{Sn}_{0.7}\right)_{0.98} \mathrm{Sb}_{0.02}$ 粉 末铺放在其上压平，接着再铺放电极材料粉末再次 压平，之后进行 SPS 烧结(烧结压力为 $30 \mathrm{MPa}$, 保温
时间为 $10 \mathrm{~min}, \mathrm{Ni}$ 作电极时烧结温度为 $973 \mathrm{~K}$ ); 另 一种是两步烧结法: 先通过 SPS 烧结(烧结压力 30 $\mathrm{MPa}$ 、烧结温度 $973 \mathrm{~K}$ 、保温时间 $10 \mathrm{~min}$ )制备致密 的 $\mathrm{Mg}_{2.16}\left(\mathrm{Si}_{0.3} \mathrm{Sn}_{0.7}\right)_{0.98} \mathrm{Sb}_{0.02}$ 块体材料, 然后将电极材 料粉体平铺在经过处理的 $\mathrm{Mg}_{2.16}\left(\mathrm{Si}_{0.3} \mathrm{Sn}_{0.7}\right)_{0.98} \mathrm{Sb}_{0.02}$ 块 体上下表面压平，再通过 SPS 烧结实现两者之间的 连接。其烧结压力为 $30 \mathrm{MPa}$, 保温时间为 5 20 min。 $\mathrm{Cu} 、 \mathrm{Ag}$ 和 $\mathrm{Al}$ 作电极的烧结温度分别为 $923 、 793$ 和 $713 \mathrm{~K}$ 。

物相组成采用荷兰 Philips 公司生产的 PANalytical X'Pert Pro 型 X 射线衍射仪进行分析, 结 合界面微观结构及成分采用日本电子珠式会社生产 的 JXA-8230 型电子显微探针分析仪(Electron probe micro-analysis, EPMA)进行表征。热膨胀系数 ( $(l)$ 采用德 国 NETZSCH 生产的热膨胀仪(Netzsch DIL402C)测量, 热扩散系数 $\lambda$ 采用德国 NETZSCH 生产的激光热导仪 (Netzsch LFA 457)测试, 热容 $\left(C_{\mathrm{p}}\right)$ 采用差式扫描量热 仪 (TA Instruments Q20) 测试，材料密度 $(d)$ 用 Archimedes 排水法测得, 热导率 $(\kappa)$ 根据公式 $\kappa=\lambda C_{\mathrm{p}} d$ 求得。材料的电导率 $\sigma$ 采用日本真空理工珠式会社生 产的 ZEM-3 型热电性能测试仪测量。

\section{2 结果与讨论}

\section{1 连接工艺}

\subsection{1一步 SPS 烧结法}

图 1 为一步 SPS 烧结法连接的 $\mathrm{Ni}$ 电极与 $\mathrm{Mg}-\mathrm{Si}-\mathrm{Sn}$ 基材料结合界面的微观形貌及成分分布 结果。在 $\mathrm{Ni}$ 电极与 $\mathrm{Mg}-\mathrm{Si}-\mathrm{Sn}$ 基材料之间有一厚度 为 $70 \mu \mathrm{m}$ 的中间层如图 1(a,b)所示, 通过 EPMA 线 扫描的成分分布图 $1(\mathrm{~d}, \mathrm{e})$ 可看出, 该中间层既包含 $\mathrm{Mg} 、 \mathrm{Si}$ 和 $\mathrm{Sn}$ 元素, 也包含 $\mathrm{Ni}$ 元素。这可能是因为 两种粉末在冷压成块体时有一定的渗透, 在 SPS 烧 结时又出现元素的互扩散。但研究结果表明 ${ }^{[12,16]}, \mathrm{Ni}$ 与 $\mathrm{Mg}_{2} \mathrm{Si}$ 的互扩散并不明显, 所以该中间层的形成 可能是因为两种粉末在冷压成块体时有一定的渗透, 形成了 $\mathrm{Ni}$ 与 $\mathrm{Mg}-\mathrm{Si}-\mathrm{Sn}$ 基材料的混合区，从而在 SPS 烧结后保留下来。

从图 1(a c)还可以看出, 无论是在界面处还是 $\mathrm{Mg}-\mathrm{Si}-\mathrm{Sn}$ 基材料内部都存在一些微裂纹, 这可能与 $\mathrm{Ni}$ 和 $\mathrm{Mg}-\mathrm{Si}-\mathrm{Sn}$ 基材料的热膨胀系数差异有关, 在 $400 \mathrm{~K} \sim 850 \mathrm{~K}$ 范围内金属 $\mathrm{Ni}$ 的热膨胀系数约为 $15 \times$ $10^{-6} \mathrm{~K}^{-1}$, 而 $\mathrm{Mg}$-Si-Sn 基材料的热膨胀系数均在 $(18 \sim$ $20) \times 10^{-6} \mathrm{~K}^{-1}$ 范围内(见图 2), 两者热膨胀系数的差 异会导致 SPS 烧结后降温过程中在 Ni 与 $\mathrm{Mg}-\mathrm{Si}-\mathrm{Sn}$ 

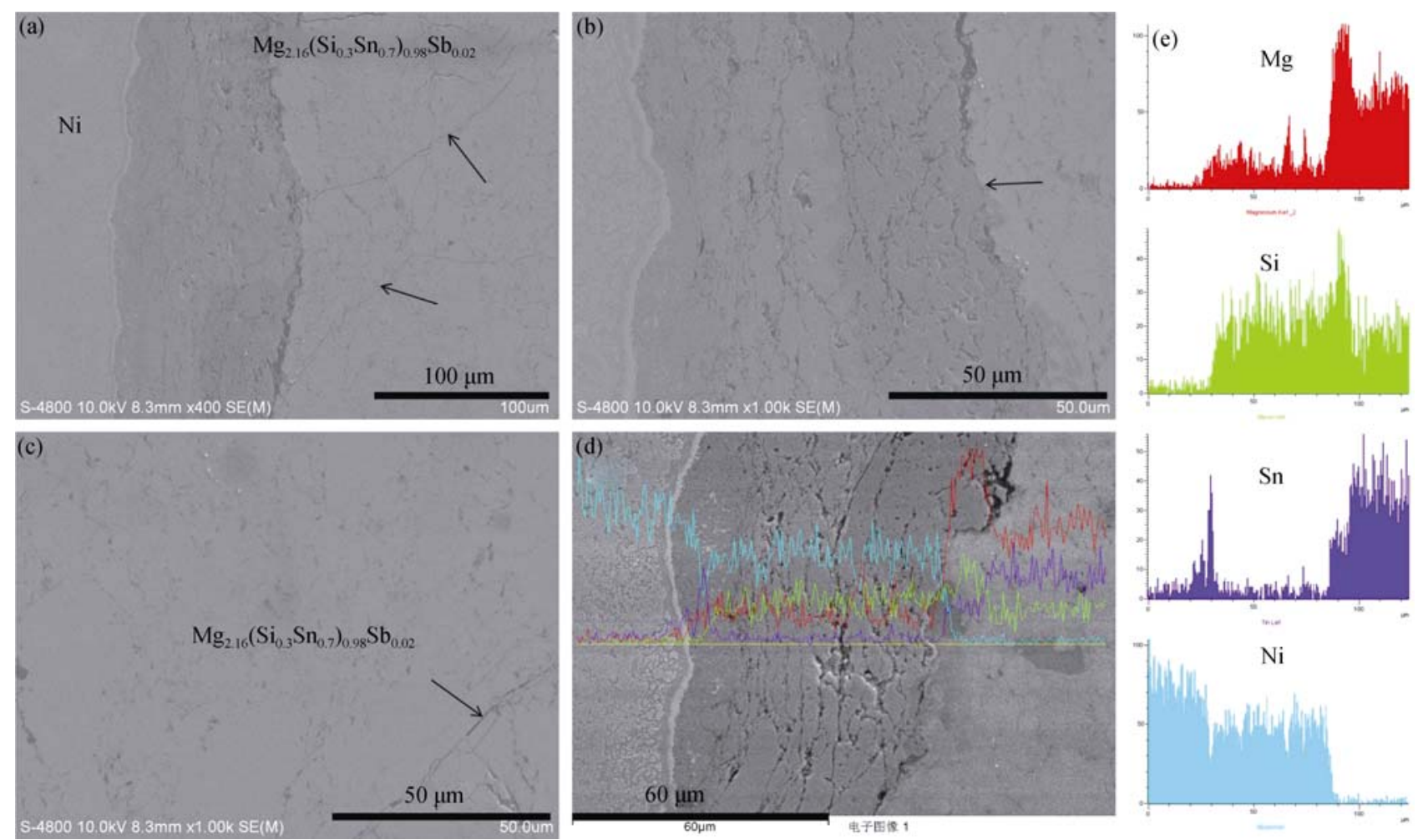

图 $1 \mathrm{Ni}$ 电极与 $\mathrm{Mg}-\mathrm{Si}-\mathrm{Sn}$ 基材料结合界面背散射和线扫描成分分布图

Fig. 1 Back scattering images and line profiles of the interface between Ni and Mg-Si-Sn by EMPA (a-b) BSE photographs of the joint between Ni and Mg-Si-Sn; (c) BSE photographs of Mg-Si-Sn; (d-e) Line profiles of the joint Arrows point to the cracks

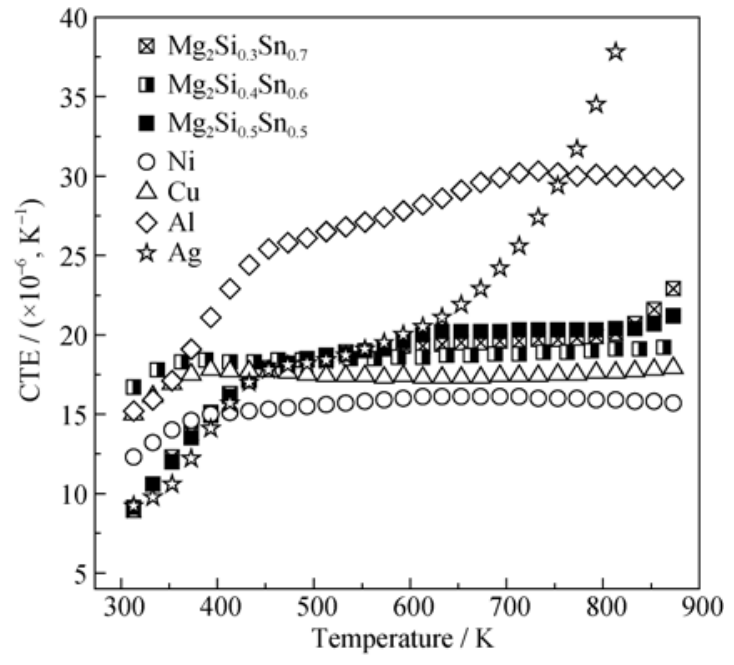

图 2 金属电极和 $\mathrm{Mg}-\mathrm{Si}-\mathrm{Sn}$ 基材料的热膨胀系数(CTE)随温 度的变化关系

Fig. 2 Temperature dependence of CTE for metal electrodes and $\mathrm{Mg}-\mathrm{Si}-\mathrm{Sn}$

材料的结合界面处产生热应力，应力集中导致微裂 纹的出现。这种微裂纹的存在会增大电极与材料之 间的接触电阻, 并影响到热电器件的转换效率。此 外, Nemoto 等 ${ }^{[16]}$ 也发现 $\mathrm{Mg}_{2} \mathrm{Si}$ 与 $\mathrm{Ni}$ 电极结合界面 之间的接触电阻较大, 所以 $\mathrm{Ni}$ 不是理想的 $\mathrm{Mg}-\mathrm{Si}-\mathrm{Sn}$ 基材料的电极材料。
采用一步 SPS 烧结法进行了其它金属单质 Al、 $\mathrm{Cu} 、 \mathrm{Ag}$ 与 $\mathrm{Mg}-\mathrm{Si}-\mathrm{Sn}$ 基材料的连接, 但实验结果表 明: 当 $\mathrm{Cu} 、 \mathrm{Ag}$ 作电极时, $\mathrm{Mg}-\mathrm{Si}-\mathrm{Sn}$ 基材料内部很容 易出现裂纹(如图 3(a,b)); 当 $\mathrm{Al}$ 作电极时, $\mathrm{Al}$ 的熔点 为 $933 \mathrm{~K}$, 为了防止 $\mathrm{Al}$ 在烧结时熔化, 实验结果表 明烧结温度不能高于 $853 \mathrm{~K}$, 大大低于 $\mathrm{Mg}-\mathrm{Si}-\mathrm{Sn}$ 基 材料的烧结温度 $973 \mathrm{~K}$, 致使 $\mathrm{Mg}-\mathrm{Si}-\mathrm{Sn}$ 基材料烧结 块体不致密(如图 3(c)), 由此可见一步 SPS 烧结法不 能很好地实现电极材料与 $\mathrm{Mg}-\mathrm{Si}-\mathrm{Sn}$ 基材料的连接。

\subsection{2 两步 SPS 烧结法}

图 4 图 6 分别为采用两步 SPS 烧结法制备的 $\mathrm{Mg}-\mathrm{Si}-\mathrm{Sn}$ 基材料与 $\mathrm{Cu}$ 电极、 $\mathrm{Ag}$ 电极和 $\mathrm{Al}$ 电极结 合界面的形貌及成分分布图。

从图 4(a,b,d,e)可观察到界面处有中间层的形成, 而且随着保温时间的延长, 各元素的互扩散增强 (图 4(c,f)), 中间层厚度逐渐变大，由 20 30 $\mu \mathrm{m}$ (保 温时间为 $10 \mathrm{~min}$, 图 4(a c ) ) 增加到约 $40 \mu \mathrm{m}$ (保温时 间为 $20 \mathrm{~min}$, 图 4(d f))。图 4(a)的插图中界面处有 非常明显的裂纹, 而在图 4(a)和图 4(d)中并未发现 明显的微裂纹, 所以 SPS 烧结后采取缓慢降温的方 式有利于减少甚至避免界面处微裂纹的产生。从本 实验的结果来看, 若能通过某种方式抑制 $\mathrm{Cu}$ 与 $\mathrm{Mg}-\mathrm{Si}-\mathrm{Sn}$ 基材料的互扩散, $\mathrm{Cu}$ 有可能是较合适的电 

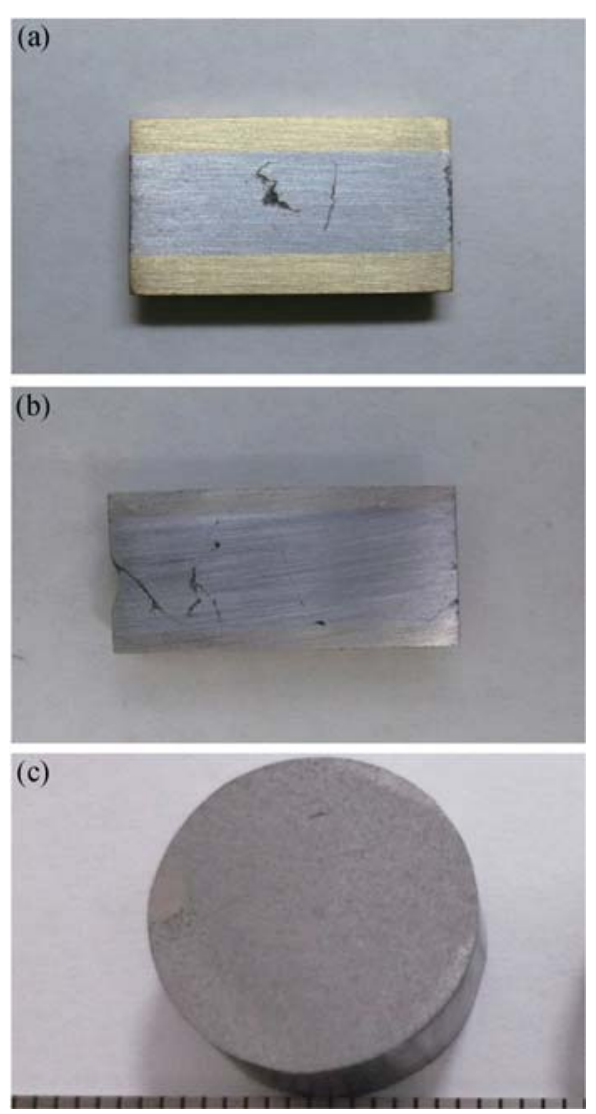

图 3 一步法烧结的电极材料与 $\mathrm{Mg}-\mathrm{Si}-\mathrm{Sn}$ 基材料样品照片

Fig. 3 Pictures of samples sintered by one step SPS

(a) Sectional view of $\mathrm{Cu} / \mathrm{Mg}-\mathrm{Si}-\mathrm{Sn}$; (b) Sectional view of $\mathrm{Ag} / \mathrm{Mg}$-Si$\mathrm{Sn}$; (c) Mg-Si-Sn of Al/Mg-Si-Sn
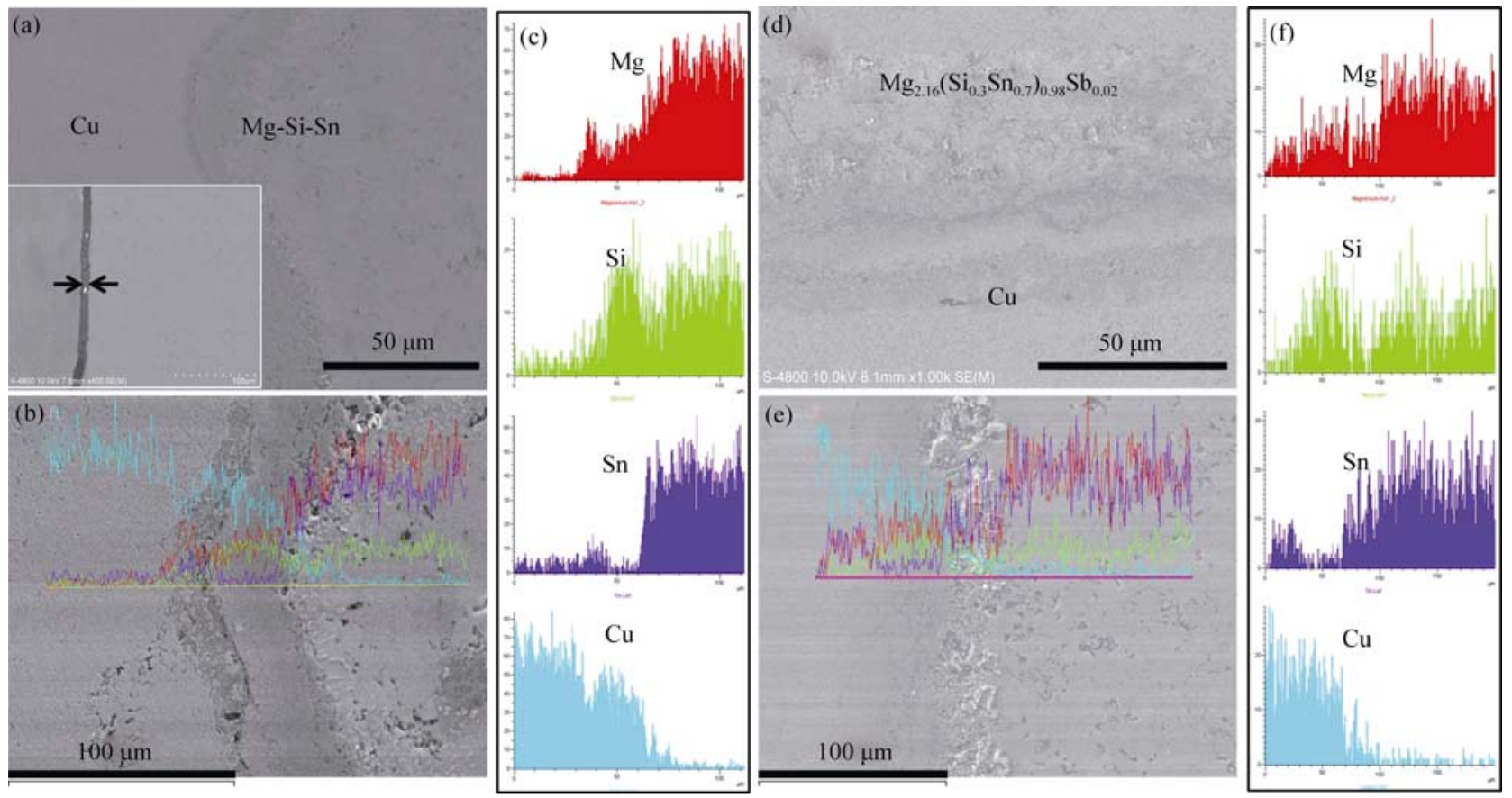

图 $4 \mathrm{Cu}$ 电极与 $\mathrm{Mg}-\mathrm{Si}-\mathrm{Sn}$ 基材料结合界面背散射和线扫描成分分布图

Fig. 4 Back scattering images and line profiles of the interface between $\mathrm{Cu}$ and $\mathrm{Mg}$-Si-Sn by EMPA

(a-c) Holding for $10 \mathrm{~min}$ and with cooling rate of $20 \mathrm{~K} / \mathrm{min}$; (a) BSE photograph; (b-c) line profiles; Inset photograph in (a) is BSE photograph of the joint with rapid cooling process; (d-f) Holding for $20 \mathrm{~min}$ and with cooling rate of $10 \mathrm{~K} / \mathrm{min}$; (d) BSE photograph; (e-f) line profiles 

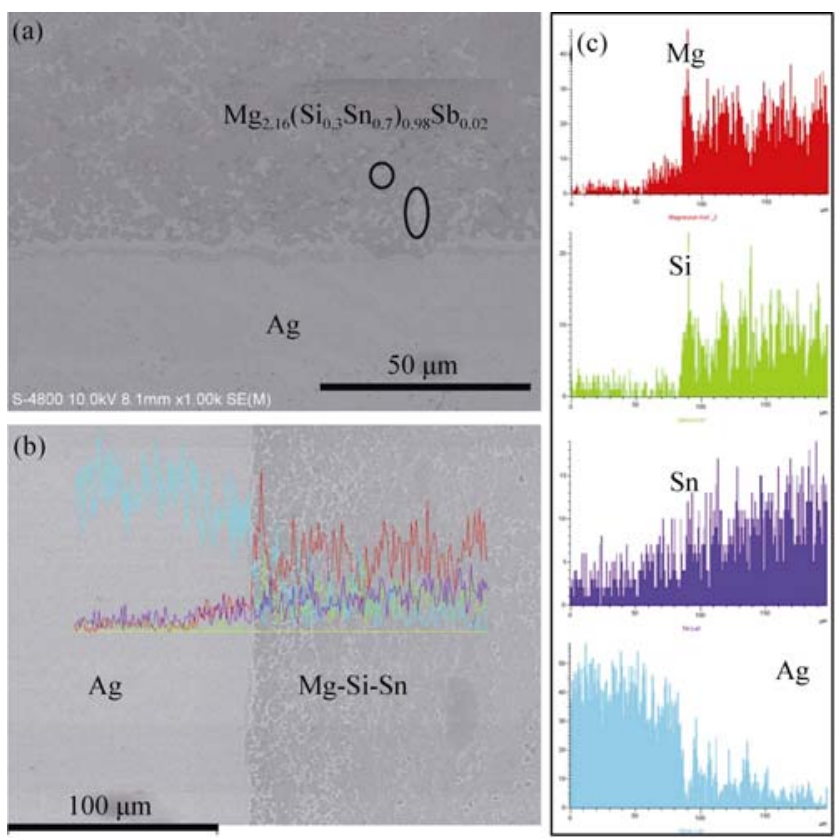

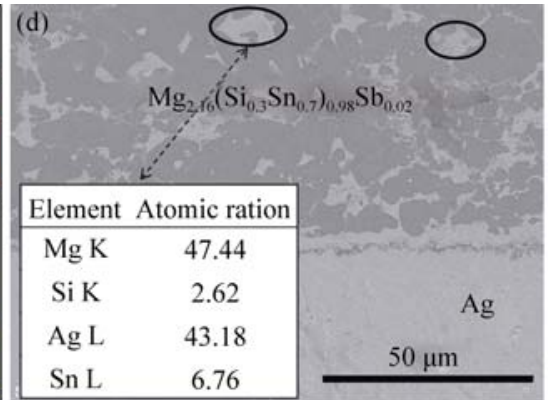

(e)

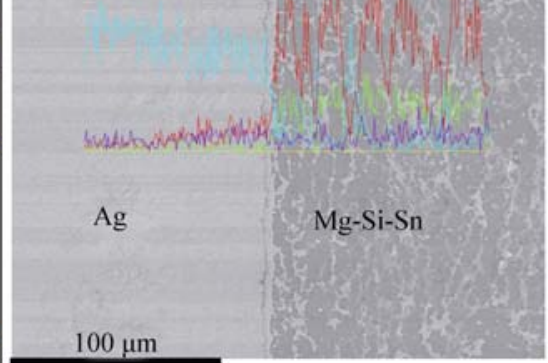

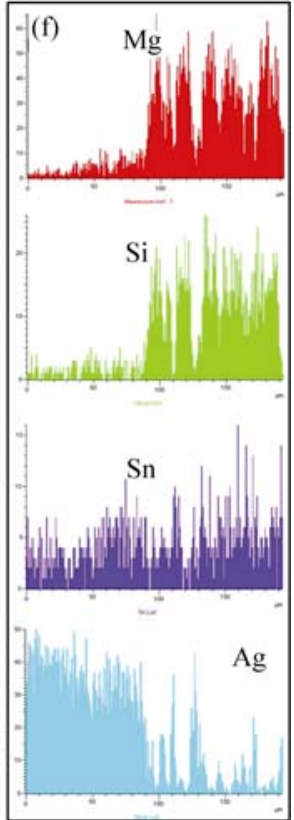

图 $5 \mathrm{Ag}$ 电极与 $\mathrm{Mg}-\mathrm{Si}-\mathrm{Sn}$ 基材料结合界面背散射和线扫描成分分布图

Fig. 5 Back scattering images and line profiles of the interface between $\mathrm{Ag}$ and $\mathrm{Mg}$-Si-Sn by EMPA (a-c) Holding for $5 \mathrm{~min}$ with cooling rate of $20 \mathrm{~K} / \mathrm{min}$; (a) BSE photograph; (b-c) Line profiles; Circles represent the region of $\mathrm{Ag}$ and $\mathrm{Mg}$ rich area; (d-f) Holding for $10 \mathrm{~min}$ with cooling rate of $10 \mathrm{~K} / \mathrm{min}$; (d) BSE photograph; (e-f) Line profiles

图 6 为 $\mathrm{Mg}-\mathrm{Si}-\mathrm{Sn}$ 基材料与 $\mathrm{Al}$ 粉末电极在不同 工艺下进行 SPS 烧结的分析结果，在图 6(a,d)中无 论是界面处或是材料内部均未发现明显的微裂纹, 结合界面干净平整。从图 6(b、c)和图 6(e、f)线扫 描成分分布图中可以看到, $\mathrm{Al}$ 与 $\mathrm{Mg} 、 \mathrm{Si} 、 \mathrm{Sn}$ 元素之
间几乎不存在互扩散，因此 $\mathrm{Al}$ 可能是较合适的 $\mathrm{Mg}-\mathrm{Si}-\mathrm{Sn}$ 基材料的电极材料。

从上面的实验分析可知，采用两步 SPS 烧结 法可较好地完成 $\mathrm{Mg}-\mathrm{Si}-\mathrm{Sn}$ 基材料与电极材料的 连接。
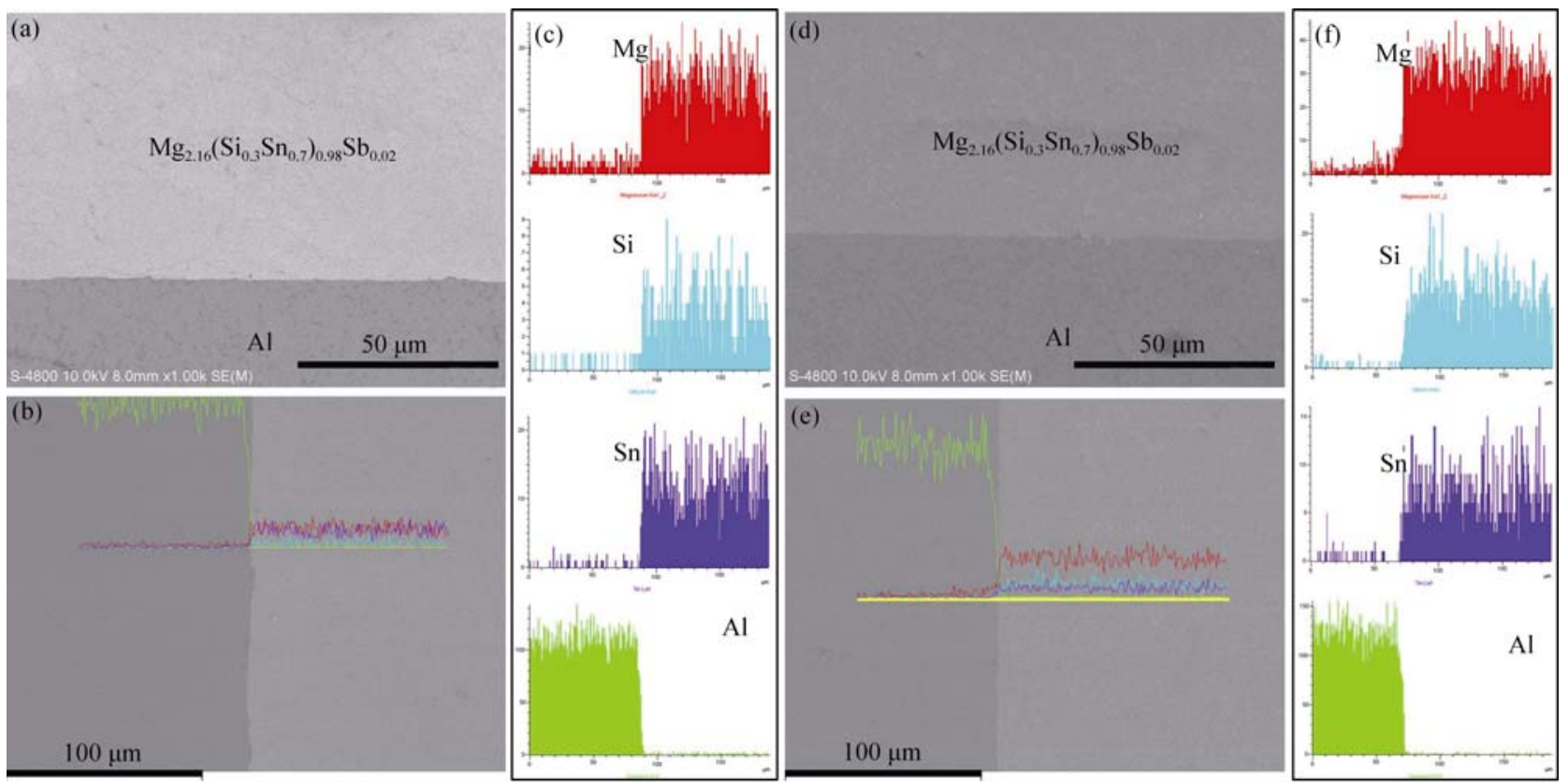

图 $6 \mathrm{Al}$ 电极与 $\mathrm{Mg}-\mathrm{Si}-\mathrm{Sn}$ 基材料结合界面背散射和线扫描成分分布图

Fig. 6 Back scattering images and line profiles of the interface between $\mathrm{Al}$ and $\mathrm{Mg}$-Si-Sn by EMPA (a-c) Holding for $5 \mathrm{~min}$ with cooling rate of $20 \mathrm{~K} / \mathrm{min}$; (a) BSE photograph; (b-c) Line profiles (d-f) Holding for $10 \mathrm{~min}$ with cooling rate of $10 \mathrm{~K} / \mathrm{min}$; (d) BSE photograph; (e-f) Line profiles 


\section{2 电极材料的优化}

依据上述实验分析结果, 尽管 $\mathrm{Cu}$ 和 $\mathrm{Al}$ 作电极 时与 Mg-Si-Sn 基材料之间扩散较少、结合界面较为 理想, 但其热膨胀系数与 $\mathrm{Mg}-\mathrm{Si}-\mathrm{Sn}$ 基材料仍存在差 异(如图 2 所示)。在热电器件长期服役过程中, 电极 材料与 $\mathrm{Mg}-\mathrm{Si}-\mathrm{Sn}$ 基热电材料之间热膨胀系数的差 异会导致两者结合界面处出现微裂纹, 严重的甚至 电极直接脱落等, 所以金属单质 $\mathrm{Cu}$ 和 $\mathrm{Al}$ 仍然不能 作为 $\mathrm{Mg}$-Si-Sn 基热电材料的电极材料。 $\mathrm{Zhao}$ 等 ${ }^{[19-22]}$ 在研究 $\mathrm{CoSb}_{3}$ 基材料的电极材料时, 为了减小所用 金属电极 $\mathrm{Cu}$ 与 $\mathrm{CoSb}_{3}$ 基材料热膨胀系数之间的差 异, 采用 $\mathrm{W}-\mathrm{Cu} 、 \mathrm{Mo}-\mathrm{Cu}$ 合金作为电极材料, 将电极 材料与 $\mathrm{CoSb}_{3}$ 基材料热膨胀系数的失配降到最低, 并且实现了两者的良好连接。考虑到 $\mathrm{Cu} 、 \mathrm{Ni}$ 的热 膨胀系数低于 $\mathrm{Mg}$-Si-Sn 基材料, 而 $\mathrm{Al}$ 的热膨胀系 数高于 $\mathrm{Mg}-\mathrm{Si}-\mathrm{Sn}$ 基材料, 本研究进一步探索了热膨 胀系数能与 $\mathrm{Mg}-\mathrm{Si}-\mathrm{Sn}$ 基材料匹配的 $\mathrm{Cu}-\mathrm{Al} 、 \mathrm{Ni}-\mathrm{Al}$
合金比例。

\subsection{1 合金电极的基本物理性能}

图 7 为购买的 $\mathrm{Cu}-\mathrm{Al}$ 合金、 $\mathrm{Ni}-\mathrm{Al}$ 合金的粉末 $\mathrm{XRD}$ 图谱, 两类合金都含有两种主相, 分别为 $\mathrm{NiAl}_{3}$ 和 $\mathrm{Ni}_{2} \mathrm{Al}_{3} 、 \mathrm{AlCu}_{3}$ 和 $\mathrm{Cu}_{9} \mathrm{Al}_{4}$ 。图 8 为合金粉末 经 SPS 烧结后得到块体的基本物性参数。尽管两类 合金都具有高的电导率和热导率，但 Ni-Al 合金的 热膨胀系数明显低于 $\mathrm{Mg}-\mathrm{Si}-\mathrm{Sn}$ 基材料, $\mathrm{Cu}-\mathrm{Al}$ 合金 的热膨胀系数稍高于 $\mathrm{Mg}$-Si-Sn 基材料(图 8), 所以 这两类合金仍然不能直接作为 $\mathrm{Mg}-\mathrm{Si}-\mathrm{Sn}$ 基材料的 电极, 要作为电极材料必需对其组分进行优化。

\subsection{2 合金电极的优化}

为了使 $\mathrm{Ni}-\mathrm{Al}$ 合金、 $\mathrm{Cu}-\mathrm{Al}$ 合金的热膨胀系数与 $\mathrm{Mg}$-Si-Sn 基材料相匹配, 尝试向合金中加入适量的 某种金属来调节其热膨胀系数, 如在 Ni-Al 合金中 加入热膨胀系数比 $\mathrm{Mg}-\mathrm{Si}-\mathrm{Sn}$ 基材料高的金属 $\mathrm{Al}$, 而在 $\mathrm{Cu}-\mathrm{Al}$ 合金中则加入热膨胀系数比 $\mathrm{Mg}-\mathrm{Si}-\mathrm{Sn}$
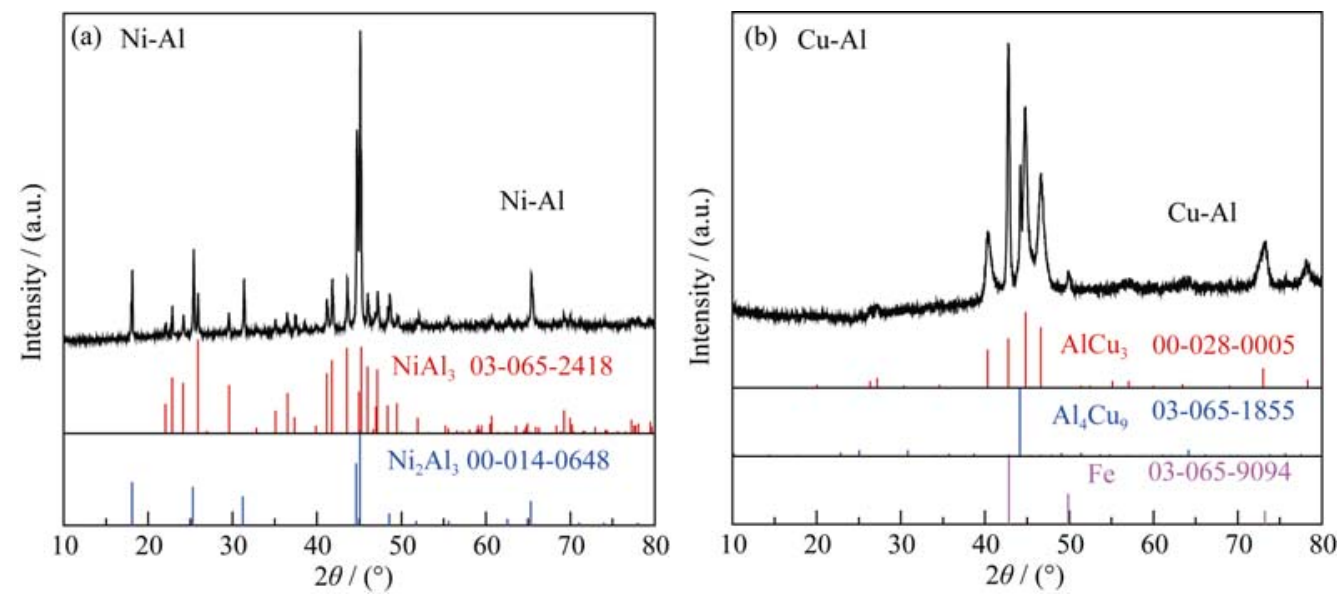

图 7 合金原料粉末的 XRD 图谱

Fig. 7 XRD patterns of raw materials.
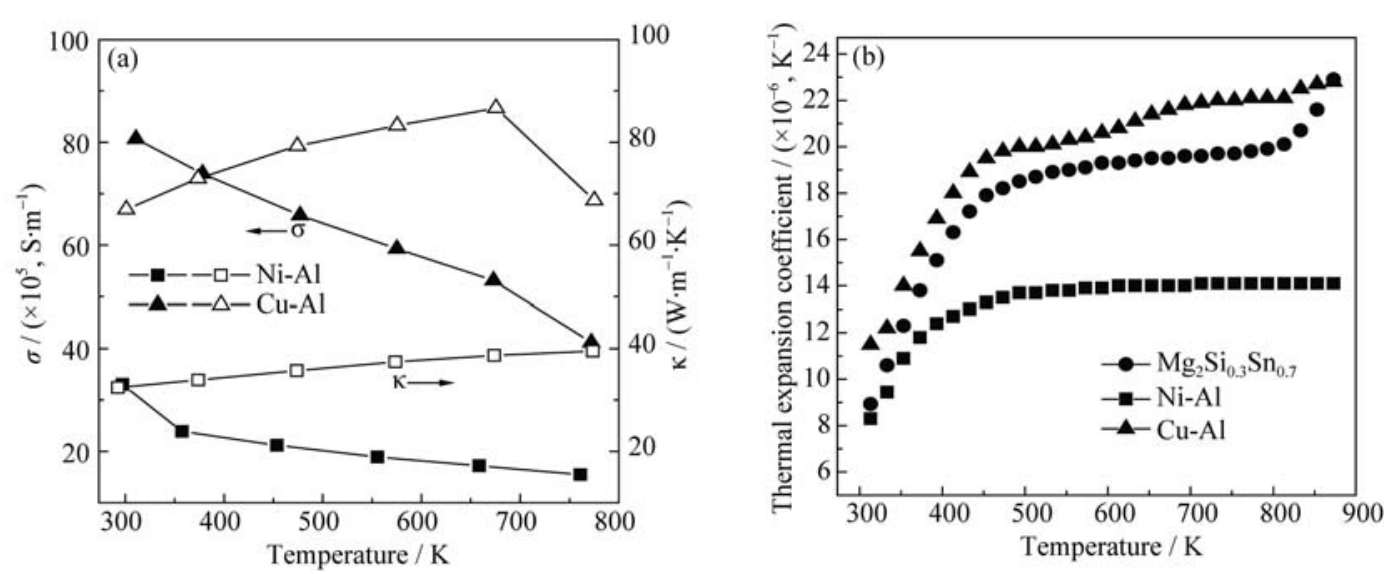

图 8 SPS 烧结合金块体的基本物性与温度关系

Fig. 8 Temperature dependence of basic physical properties for alloy material 
基材料低的金属 $\mathrm{Cu}$ 。

图 9 为 $\mathrm{Ni}-\mathrm{Al} / \mathrm{Al}$ 的复合电极材料 SPS 烧结后得 到块体的基本物理性能测试结果。在测试温度范围 内, 加入 $\mathrm{Al}$ 以后复合电极的电导率和热导率都有较 大幅度的增加, 且热膨胀系数得到了较好的调控。

当 $\mathrm{Ni}-\mathrm{Al}$ 与 $\mathrm{Al}$ 配比为 $60: 40$ 时, 在 $350 \sim 750 \mathrm{~K}$ 的温 度范围内, $\mathrm{Ni}-\mathrm{Al} / \mathrm{Al}$ 复合电极与 $\mathrm{Mg}-\mathrm{Si}-\mathrm{Sn}$ 基热电材 料的热膨胀系数较为匹配。

图 10 为 $\mathrm{Cu}-\mathrm{Al} / \mathrm{Cu}$ 复合电极材料经 SPS 烧结后 得到块体的基本物理性能测试结果。在测试温度范 围内, $\mathrm{Cu}$ 的加入提高了复合电极的电导率和热导率, 同时调控了复合电极的热膨胀系数。当 $\mathrm{Cu}-\mathrm{Al}$ 与 $\mathrm{Cu}$ 配比为 $45 ： 55$ 时, 在 $400 \sim 800 \mathrm{~K}$ 的温度范围内, 复 合电极与 $\mathrm{Mg}-\mathrm{Si}-\mathrm{Sn}$ 基热电材料的热膨胀系数较为 匹配。

\section{3 结论}

本研究探讨了单质金属 $\mathrm{Ni} 、 \mathrm{Cu} 、 \mathrm{Ag}$ 和 $\mathrm{Al}$ 作为 电极与 $\mathrm{Mg}-\mathrm{Si}-\mathrm{Sn}$ 基材料的连接工艺及结合界面的 微观形貌，分析了 $\mathrm{Al} 、 \mathrm{Cu}$ 等金属的不同加入量对
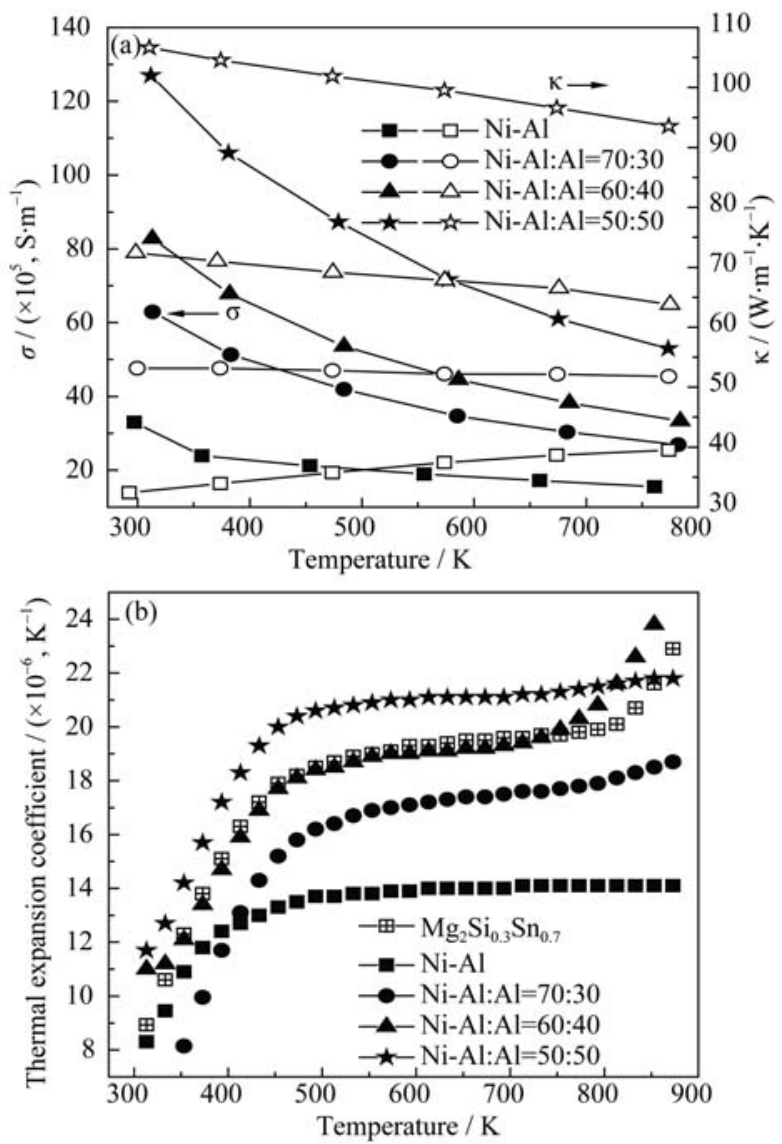

图 $9 \mathrm{Ni}-\mathrm{Al} / \mathrm{Al}$ 的基本物性随温度的变化关系

Fig. 9 Physical properties as a function of temperature for $\mathrm{Ni}-\mathrm{Al} / \mathrm{Al}$
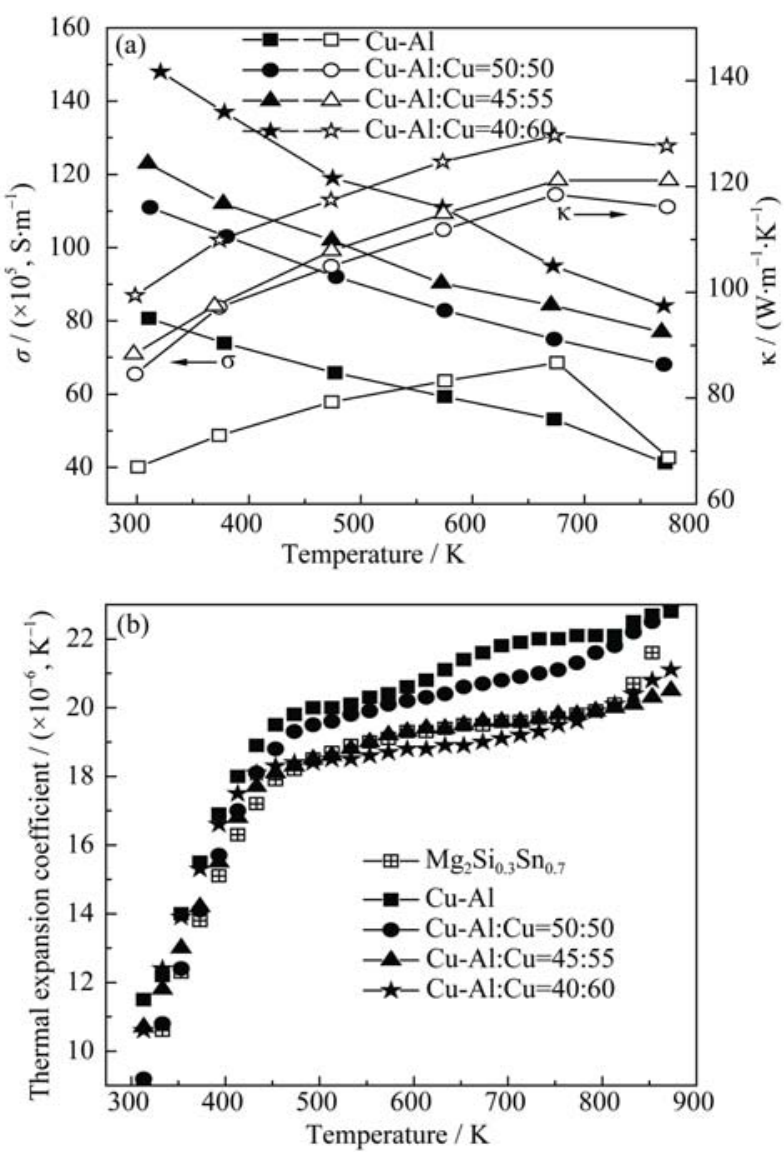

图 $10 \mathrm{Cu}-\mathrm{Al} / \mathrm{Cu}$ 的基本物性随温度的变化关系

Fig. 10 Physical properties as a function of temperature for $\mathrm{Cu}-\mathrm{Al} / \mathrm{Cu}$

$\mathrm{Ni}-\mathrm{Al} 、 \mathrm{Cu}-\mathrm{Al}$ 合金作为电极的基本物性参数的影响, 得到以下结论:

1) 采用 SPS 烧结可较好地实现 Mg-Si-Sn 基材 料与电极材料的连接，即先通过固相反应结合 SPS 制备致密的 Mg-Si-Sn 块体材料, 然后将研磨好的粉 末电极材料置于 $\mathrm{Mg}-\mathrm{Si}-\mathrm{Sn}$ 基致密块体的两端再次 进行 SPS 烧结, 烧结温度与所用电极有关, 烧结压 力为 $30 \mathrm{MPa}$, 保温时间为 $5 \sim 10 \mathrm{~min}$, 冷却速度为 $10 \sim 20 \mathrm{~K} / \mathrm{min}$ 。

2) 合金/金属的复合可实现热膨胀系数的自由 调控，同时还可以提高复合电极的电导率和热导 率。比例为 $\mathrm{Ni}-\mathrm{Al}: \mathrm{Al}=60: 40 、 \mathrm{Cu}-\mathrm{Al}: \mathrm{Cu}=45: 55$ 的复合电极与 $\mathrm{Mg}-\mathrm{Si}-\mathrm{Sn}$ 基材料的热膨胀系数较为 匹配，同时也具备足够高的电导率和热导率，有可 能成为 $\mathrm{Mg}-\mathrm{Si}-\mathrm{Sn}$ 基材料较为理想的电极材料。

参考文献:

[1] VINING C B. Chapter 23 Thermoelectric Properties of Silicides. CRC handbook of thermoelectrics. Raton London New York 
Washington, D C: CRC Press LLC, 1995: 277-285.

[2] HAN Z M, ZHANG X, LU Q M, et al. Preparation and thermoelectric properties of $\left(\mathrm{Mg}_{2} \mathrm{Si}_{1-x} \mathrm{Sb}_{x}\right)_{0.4}-\left(\mathrm{Mg}_{2} \mathrm{Sn}\right)_{0.6}$ alloys. Journal of Inorganic Materials, 2012, 27(8): 822-826.

[3] ZAITSEV V K, FEDOROV M I, GURIEVA E A, et al. Highly effective $\mathrm{Mg}_{2} \mathrm{Si}_{1-x} \mathrm{Sn}_{x}$ thermoelectrics. Physical Review B, 2006, 74(4): 045207.

[4] NODA Y, KON H, FURUKAWA Y, et al. Preparation and thermoelectric properties of $\mathrm{Mg}_{2} \mathrm{Si}_{1-x} \mathrm{Ge}_{x} \quad(x=0-0.4)$ solid solution semiconductors. Materials Transactions, JIM, 1992, 33(9): $845-850$

[5] SONG R B, LIU Y Z, AIZAWA T. Solid state synthesis and thermoelectric properties of Mg-Si-Ge system. Journal of Materials Science \& Technology, 2005, 21(5): 618-622.

[6] FEDOROV M I, ZAITSEV V K, EREMIN I S, et al. Transport properties of $\mathrm{Mg}_{2} \mathrm{X}_{0.4} \mathrm{Sn}_{0.6}$ solid solutions ( $x=\mathrm{Si}, \mathrm{Ge}$ ) with p-type conductivity. Physics of the Solid State, 2006, 48(8): 1486-1490.

[7] SAKAMOTO T, IIDA T, MATSUMOTO A, et al. Thermoelectric characteristics of a commercialized $\mathrm{Mg}_{2} \mathrm{Si}$ source doped with $\mathrm{Al}, \mathrm{Bi}$, $\mathrm{Ag}$ and Cu. Journal of Electronic Materials, 2010, 39(9): 1708-1713.

[8] LIU W, TANG X F, LI H, et al. Optimized thermoelectric properties of Sb-doped $\mathrm{Mg}_{2(1+z)} \mathrm{Si}_{0.5-\mathrm{y}} \mathrm{Sn}_{0.5} \mathrm{Sb}_{y}$ through adjustment of the Mg content. Chemistry of Materials, 2011, 23(23): 5256-5263.

[9] TAN X J, LIU W, LI H, et al. Multiscale calculations of thermoelectric properties of n-type $\mathrm{Mg}_{2} \mathrm{Si}_{1-x} \mathrm{Sn}_{x}$ solid solutions. Physical Review B, 2012, 85(20): 205212.

[10] LIU W. Optimization of Thermoelectric Properties of n-type $\mathrm{Mg}_{2} \mathrm{Si}_{1-x} \mathrm{Sn}_{x}$ Based Materials Through Doping As Well As the Adjustment and Cotrol of Band Structure. Wuhan: Wuhan University of Technology. PhD Thesis, 2012: 28.

[11] SAKAMOTO T, IIDA T, TAGUCHI Y, et al. Examination of a thermally viable structure for an unconventional uni-leg $\mathrm{Mg}_{2} \mathrm{Si}$ thermoelectric power generator. Journal of Electronic Materials, 2012, 41(6): 1429-1435.

[12] Nemoto T, IIDA T, SATO J, et al. Power generation characteristics of $\mathrm{Mg}_{2} \mathrm{Si}$ uni-leg thermoelectric generator. Journal of Electronic Materials, 2012, 41(6): 1312-1316.

[13] SAKAMOTO T, IIDA T, KUROSAKI S, et al. Thermoelectric behavior of $\mathrm{Sb}$ - and $\mathrm{Al}$-doped $\mathrm{n}$-type $\mathrm{Mg}_{2} \mathrm{Si}$ device under large temperature differences. Journal of Electronic Materials, 2011, 40(5): 629-634.

[14] SAKAMOTO T, IIDA T, FUKUSHIMA N, et al. Thermoelectric properties and power generation characteristics of sintered undoped n-type $\mathrm{Mg}_{2} \mathrm{Si}$. Thin Solid Films, 2011, 519(24): $8528-8531$.

[15] SAKAMOTO T, IIDA T, HONDA Y, et al. The use of ransition-metal silicides to reduce the contact resistance between the lectrode and sintered n-type $\mathrm{Mg}_{2} \mathrm{Si}$. Journal of Electronic aterials, 2012, 41(6): 1805-1810.

[16] NEMOTO T, IIDA T, SATO J, et al. Characteristics of a pin-fin structure thermoelectric uni-leg device using a commercial n-type $\mathrm{Mg}_{2} \mathrm{Si}$ source. Journal of Electronic Materials, 2010, 39(9): 1572-1578.

[17] FAN J F, CHEN L D, BAI S Q et al. Joining of Mo to $\mathrm{CoSb}_{3}$ by spark plasma sintering by inserting a Ti interlayer. Materials Letters, 2004, 58: 3876-3878.

[18] WOJCIECHOWSKI K T, ZYBALA R, MANIA R. High temperature $\mathrm{CoSb}_{3}-\mathrm{Cu}$ junctions. Microelectronics Reliability, 2011, 51(7): 1198-1202.

[19] ZHAO D G, GENG H R, TENG X Y. Fabrication and reliability evaluation of $\mathrm{CoSb}_{3} / \mathrm{W}-\mathrm{Cu}$ thermoelectric element. Journal of Alloys and Compounds, 2012, 517: 198-203.

[20] ZHAO D G, LI X Y, HE L, et al. High temperature reliability evaluation of $\mathrm{CoSb}_{3} /$ electrode thermoelectric joints. Intermetallics, 2009, 17(3): 136-141.

[21] ZHAO D G, LI X Y, HE L, et al. Interfacial evolution behavior and reliability evaluation of $\mathrm{Cosb}_{3} / \mathrm{Ti} / \mathrm{Mo}-\mathrm{Cu}$ thermoelectric joints during accelerated thermal aging. Journal of Alloys and Compounds, 2009, 477(1/2): 425-431.

[22] ZHAO D G, Li X Y, JIANG W, et al. Fabrication of $\mathrm{CoSb}_{3} / \mathrm{MoCu}$ thermoelectric joint by one-step SPS and evaluation. Journal of Inorganic Materials, 2009, 24(3): 545-548. 